\title{
seal of approval program for radio and television weathercasting
}

\section{The program as of $1988^{1}$}

The goal of the Seal of Approval program is to continuously upgrade radio and television weather programs. Since the weathercaster is the primary representative of the meteorological profession to the public, we, as meteorologists, have a responsibility in helping recognize those who are competent.

The Board of Broadcast Meteorology ${ }^{1}$ was formed by the Council in 1957 to establish criteria for the evaluation of weathercasts and to conduct the evaluation procedure. The growth of television and radio has been accompanied by an increasing impact on the public; therefore, the need for professional weathercasting and its recognition by the AMS have been quite apparent.

Evaluation procedures were established and the first television kinescope recordings and radio tapes were evaluated by the Board in late 1959. The first Seals of Approval, other than for Board members, were granted in January 1960.

Seals have been awarded to weathercasters with widely varied approaches to presenting the weather on television; some of these can be seen in an hour-long film, Weathercasting, Coast to Coast, which is available from the AMS for a two-day rental fee of $\$ 75$.

It has always been the goal of the Seal of Approval program to foster high standards of professionalism among broadcast meteorologists. Recently, however, the courts have attacked the use of the term "professionalism" as being overbroad and capable of misuse. Indeed, this standard has many different meanings to different individuals. We have produced a more specific definition. Henceforth the stated goals of the program will be to insure that meteorologists who hold the Seal of Approval exhibit scientific competence and effective communication skills in their weather forecasts.

\section{Applications}

A. Upon request from any individual, the Secretary-Treasurer of the Society will furnish an application form. Applications will be accepted only from full members of the AMS or those nonmembers who meet all qualifications for membership, as defined in the Constitution of the Society.

B. The application form must be completed and returned to the Secretary-Treasurer along with the appropriate application fee. The fee for television is $\$ 150$ for members and $\$ 360$ for nonmembers, including the BULLETIN OF THE AMS. The fee for radio is $\$ 100$ for members and $\$ 260$ for nonmembers, including the BULLETIN OF THE AMS.

\footnotetext{
${ }^{1}$ The Board is currently made up of: Valerie Voss, Cable News Network, Atlanta, GA, Chairman; Vincent D. Condella, Jr., WITI-TV Weather, Milwaukee, WI; Allan C. Eustis, WJLA-TV, Washington, DC; Gary W. Ley, WJAR-TV, Providence, RI; Mike Nelson, KMOV-TV, St. Louis, MO; Elliot Abrams, Accu-Weather Inc., State College, PA; Paul Gross, WDIV-TV, Detroit, MI.
}

C. The Secretary-Treasurer then forwards the application to the Chairman of the Board of Broadcast Meteorology.

\section{Selection of Evaluating Committee}

A. The Board Chairman will select five members of the Board who will constitute the reviewing panel for that applicant. Each member of the panel shall certify to the Chairman that he or she is aware of no conflict of interest in accepting the appointment to review any particular applicant. The applicant will be notified in writing of the names and occupations of the members selected and will be given an opportunity to object to any of the Board members selected. An applicant shall be required to state in writing the reasons for any objection to any member of the panel. The Chairman may either accept the objection from the applicant or refer the question to the Chairman of the Commission on Professional Affairs for final decision; the Commissioner shall act on the preponderance of the evidence. All applicants shall be notified that unless objection is received within 15 days, the Chairman will assume the selected Board members are satisfactory to the applicant and will proceed to the next step.

\section{Submission of Evaluation Materials}

A. The Chairman will then request that the applicant submit three video or audio tape examples of his or her work. These examples must be of broadcasts made on three consecutive appearance days. In the case of individuals currently employed "'on-air," the tapes shall be of actual on-air performances, including lead-ins and throw-backs to other on-air talent. All commercial messages shall be edited out of the tape. No minimum time of each segment will be required. Those not currently employed are allowed to submit equivalent simulated tapes. Performances on tapes submitted must be less than six months old as of the date of the application. Applicants must also certify that weathercasts submitted are substantially the result of his or her own work.

\section{Grading Process}

A. The applicant sends his or her tapes directly to each member of the evaluating panel. Panel members will review the tapes based on four criteria:

\section{Technical competence}

This is a two-step evaluation. First, as indicated above, all candidates must either be full members of the Society or demonstrate sufficient educational and professional background to meet the standards for membership. The second step is an evaluation of the submitted tapes to determine whether the information contained therein is scientifically and technically valid.

\section{Informational value}

This category will be used to evaluate whether the candidate has given the audience sufficient informa- 
tion about weather conditions locally and nationally in the immediate past, currently, and to be expected.

\section{Explanatory value}

Reviewers are asked to determine whether or not the candidate has given sufficient explanation of the processes which produce the recent, current, and anticipated weather conditions.

\section{Communication skills}

This criterion is intended to measure the effectiveness of the means chosen by the candidate to communicate information and explanations. Since this is a highly subjective area, only a candidate's clear failure to communicate the information will be judged as grounds for failure. Subjective judgments concerning appearance, sets, lead-ins, props, map symbols and voice quality will not be used unless cumulatively the negative effect of a poor performance in any of these areas compels the conclusion that the candidate has completely failed to communicate his or her message.

B. Grades will be awarded in each of the four categories on a scale of 4.0 , as follows:
1.0: unacceptable
2.0: substandard
2.5: satisfactory (passing)
3.0: good
3.5: excellent
4.0: superior

Each reviewer will submit a grade in each category. The grades given by all members will be averaged by the Chairman. A candidate must score at least a 2.5 average in each category in order to succeed on the examination.

\section{Notification}

A. Successful applicants will be notified by letter from the President of the Society. Station managers will also receive congratulatory letters, and press releases will be sent from Society headquarters to the major print media within the market where the applicant is employed.

B. Unsuccessful applicants will be notified by letter from the Secretary-Treasurer of the Society. The letter shall contain a statement from the Chairman of the Board, explaining the failure and suggesting ways of improving the performance based upon the information received from panel members. Unsuccessful applicants may reapply after a period of three months from the date of the negative notification. The reapplication fee is $\$ 50$. After a second unsuccessful application, there will be a one-year waiting period before a third application can be submitted.

\section{Miscellaneous}

A. Successful candidates may move their Seal from station to station without further application to the Society. However, relocation from a national (for example, $\mathrm{CNN}$ or The Weather Channel) to a local appearance requires the submission of new tapes (but no additional fees) for review by the Board in accord with the four stated criteria.
B. Seals may be renewed annually by submitting a renewal fee and attesting that the overall content and format of on-air presentations have not changed significantly. The annual renewal fee for members is $\$ 20$, and for nonmembers, $\$ 100$.

C. Successful candidates will be informed of their right to use the Seal and of any limitations which the Society may impose upon such use.

D. Unsuccessful candidates may appeal the negative decision of the Board of Broadcast Meteorology to the Executive Committee of the Society within 90 days of the date of notification.

\section{Suspension or Revocation}

A. The American Meteorological Society reserves the right to suspend or revoke the right to use the Seal if a seal holder, in the conduct of his profession, clearly fails to conduct himself in a manner that reflects the dignity and honor of the profession or if a seal holder fails repeatedly to adhere to the four criteria for the award of the Seal set out in Section IV.

B. Any complaint that may be grounds for suspension or revocation of the Seal under Paragraph A shall be sent to the Secretary-Treasurer of the Society. The SecretaryTreasurer may summarily dismiss a frivolous complaint with notice and a statement of reasons to the complainant. Otherwise, the Secretary-Treasurer shall send a copy of the complaint to the Chairman of the Commission on Professional Affairs, the Chairman of the Board of Broadcast Meteorology, and the seal holder.

C. The Chairman of the Board of Broadcast Meteorology may resolve any complaint by any appropriate informal means, which may include contacting the seal holder in question in an effort to resolve by mutual agreement the subject of the complaint.

D. Any complaint not disposed of by informal means shall be determined as follows:

1. With the concurrence of the Chairman of the Commission on Professional Affairs, a copy of the complaint shall be sent to the seal holder in question by the Board Chairman, together with a copy of this Section VII constituting the procedure for suspension or revocation of the Seal of Approval and the names and brief descriptions of the members of a Fact-Finding Panel appointed under Sub-Paragraph 2.

2. The Board Chairman shall select three members of the Board who will constitute a Fact-Finding Panel to determine the issues raised in the complaint. Each member of the Fact-Finding Panel shall certify to the Chairman that he or she is aware of no conflict of interest in accepting the appointment to the Fact-Finding Panel. The seal holder in question shall have the opportunity within fifteen (15) days of the date of notification to object to any member of the Fact-Finding Panel. The seal holder shall be required to state in writing the reasons for any objection to a member of the panel. The Chairman may either accept the 
objection from the seal holder or refer the question to the Chairman of the Commission on Professional Affairs for final decision.

3. The seal holder shall cooperate fully with the FactFinding Panel and produce any tangible information relevant to the issues raised in the complaint and shall attempt to secure for the Fact-Finding Panel copies or recordings of any weathercast in issue. The seal holder may submit to the Fact-Finding Panel any other information he or she deems relevant, including copies or recordings of other weathercasts and a written answer to the complaint.

4. After the submission of the tangible-evidence to the Fact-Finding Panel, if any facts remain in dispute, and upon the written request of the seal holder, a hearing shall be held by the Fact-Finding Panel upon adequate notice to the seal holder and at a time and place convenient to the members of the panel and the seal holder. The hearing shall be conducted according to fundamental concepts of due process recognized as fair and followed by administrative agencies in the conduct of adjudicatory hearings, which shall include the right to counsel, presentation of witnesses, right to cross-examination, and the right to record the proceeding by either stenographic or tape recording means. Strict rules of evidence shall not apply, but the panel shall accept information or evidence that is customarily relied upon by reasonable people in the conduct of serious affairs.

5. The Fact-Finding Panel shall make written findings of fact and shall determine if the seal holder has, in the conduct of his profession, clearly failed to conduct himself in a manner that reflects the dignity and honor of the profession, or if the seal holder has failed repeatedly to adhere to the four criteria for the award of the Seal as set out in Section IV. If the Fact-Finding Panel determines that the seal holder has failed in his conduct or adherence to the criteria as aforesaid, then the Fact-Finding Panel shall include in its written decision findings on the degree of severity of the matter and a recommendation for the imposition of sanctions, which may include:

a) A written informal admonition by the Chairman of the Commission on Professional Affairs, a copy of which shall be retained in the seal holder's personal file; or

b) A suspension of the seal holder's right to use the Seal of Approval for a period of time; or

c) A revocation of the seal holder's Seal of Approval.

6. The written decision of the Fact-Finding Panel shall be sent to the Chairman of the Board of Broadcast Meteorology, the Chairman of the Commission on Professional Affairs, and the seal holder. The Chairman of the Board of Broadcast Meteorology and the Chairman of the Commission on Professional Affairs, after a review of the decision, shall jointly determine in their judgment the appropriate sanction and administer the same.

E. A seal holder against whom action has been taken above may, within thirty (30) days of the date of notification of the action, appeal the action in writing to the Executive Committee of the Society.

\section{Radio Seals of Approval (Alphabetical List of Active Seal Holders)}

\author{
Abrams, Elliot \\ Akemann, Sharon L. \\ Austin, Harold S., Jr. \\ Babikian, Gary \\ Ban, Raymond J. \\ Borawski, Kathleen \\ Chan, Peter V. \\ Dahl, David W. \\ Dandrea, Joseph A. \\ Davis, Francis K., Jr. \\ Dooley, John C. \\ Eichorn, David N. \\ Endersen, G. William \\ Eustis, Allan C. \\ Falconer, Raymond E. \\ Fidler, James C. \\ Filloon, Karen A. \\ Finckle, Earl S. \\ Fishel, Gregory B. \\ Gertz, Charles M. \\ Geyer, James R. \\ Glickman, Todd S. \\ Grueber, Eugene C.
}

Head, Paul A.

Horstmeyer, Steven L.

Jaggers, James W.

Joseph, Paul S.

Kimmel, Troy M., Jr.

Kinnan, Henry W.

Lynch, Michael W.

Madson, Michael J.

Magnuson, Thomas L.

Mahar, Lawrence R.

Maly, Daniel D.

McKinley, Kenneth C.

McLain, Robert W.

McMurray, John T.

Mercer, Jack M.

Muzio, Miles S.

Myers, Joel N.

Norman, Royal L., Jr.

Peterson, William D.

Phillips, Edwin A.

Randby, William E.

Rao, Joseph M.

Rao, Renate M. 
Riggs, Jim F.

Rubin, Eugene M.

Schulz, Kenneth A.

Slesick, Leonard M.
Story, Gregory J.

Trenda, Ronald W.

Weiner, Craig A.

Williams, Kevin D.
86

91

106

93

Radio Seal Holders (Listed by Certificate Number)

\begin{tabular}{|c|c|c|c|}
\hline 1 & Francis K. Davis, Jr., Honorary & January & 1959 \\
\hline 2 & Kenneth H. Jehn*, & January & 1959 \\
\hline 3 & Henry W. Kinnan, & January & 1959 \\
\hline 4 & Milton F. Barlow** & January & 1960 \\
\hline 5 & Albert E. Boyer, Jr.** & January & 1960 \\
\hline 6 & Robert L. Hendrick** & January & 1960 \\
\hline 7 & George M. Howe** & January & 1960 \\
\hline 8 & James M. Macdonald, Jr.** & January & 1960 \\
\hline 9 & Frederick P. Ostby, Jr.** & January & 1960 \\
\hline 10 & Howard M. Frazier** & October & 1960 \\
\hline 11 & Robert G. Beebe** & October & 1960 \\
\hline 12 & Howard H. Hanks, Jr.* & October & 1960 \\
\hline 13 & James C. Fidler, & January & 1961 \\
\hline 14 & Lawrence R. Mahar & January & 1961 \\
\hline 15 & Eugene C. Grueber & August & 1961 \\
\hline 16 & Norman F. Lacey** & May & 1963 \\
\hline 17 & Alan F. Jones** & May & 1963 \\
\hline 18 & Conrad B. Gosset** & April & 1964 \\
\hline 19 & Raymond E. Falconer & July & 1964 \\
\hline 20 & Albert W. Duckworth** & September & 1964 \\
\hline 21 & William J. Hartnett** & October & 1964 \\
\hline 22 & J. R. Dixon* & October & 1964 \\
\hline 23 & Armand R. Iaccheo** & October & 1964 \\
\hline 24 & Stuart P. Marr** & October & 1964 \\
\hline 25 & John A. Ebert** & March & 1965 \\
\hline 26 & Ray A. Cathcart** & March & 1965 \\
\hline 27 & Harry C. Zimmerman** & July & 1965 \\
\hline 28 & Thomas W. Spence** & November & 1965 \\
\hline 29 & John W. Hambleton** & July & 1966 \\
\hline 30 & Seth D. Kemble** & February & 1967 \\
\hline 31 & John H. Erikson** & February & 1967 \\
\hline 32 & Kenneth C. Garee*** & February & 1967 \\
\hline 33 & Robert F. Zames** & February & 1967 \\
\hline 34 & William B. Hovey** & April & 1967 \\
\hline 35 & Conrad L. Johnson** & April & 1967 \\
\hline 36 & Frederick J. Norman** & September & 1967 \\
\hline 37 & Leonard M. Slesick & November & 1967 \\
\hline 38 & Walter Devanas** & November & 1967 \\
\hline 39 & Jehu D. Ashmore** & August & 1969 \\
\hline 40 & John T. Ghiorse, Jr.** & October & 1969 \\
\hline 41 & Charles M. Umpenhour** & December & 1969 \\
\hline 42 & Sidney O. Barnard** & March & 1970 \\
\hline 43 & Robert W. McLain & September & 1970 \\
\hline 44 & Thomas G. Wills** & June & 1971 \\
\hline 45 & Walter A. Lyons** & July & 1971 \\
\hline 46 & Paul S. Joseph & July & 1971 \\
\hline
\end{tabular}

${ }^{*}$ Deceased

** Inactive
47 William N. Seiler**

July

October 1971

48 W. Paul Catoe**

49 George R. Wooten, Jr.**

50 Earl S. Finckle

51 Elliot Abrams

52 Joel N. Myers

53 George N. Brancato**

54 Robert K. Ryan**

55 Lyle E. Brosche**

56 Allan C. Eustis

57 J. Michael Fritsch**

58 John F. Henz**

59 Vincent R. Scheetz**

60 Virginia J. Bigler-Engler**

61 John T. McMurray

62 Malcolm P. Sillars**

63 Daniel D. Maly

64 Steven D. Hilberg**

65 Steven A. Root**

66 John R. Stansfield**

67 Todd Gross**

68 Jack M. Mercer

69 Craig A. Johnson**

70 Charles M. Gertz

71 Todd S. Glickman

72 William D. Peterson

73 Eugene M. Rubin

74 Raymond J. Ban

75 Gerard K. Butch**

76 Steven L. Horstmeyer

77 William O. Alexander**

78 Howard K. Bryant**

79 Edwin A. Phillips

80 Aaron Williams, Jr.**

81 Joseph A. Dandrea

82 Thomas L. Magnuson

83 James H. Block**

84 Beverly S. V. Cudbird*

85 Donald L. Edwards**

86 Gregory J. Story

87 John C. Dooley

88 Daniel F. Smith**

89 Kathleen Borawski

90 G. William Endersen

91 Ronald W. Trenda

92 Michael W. Lynch

93 Kevin D. Williams

94 Lee C. Anderson**

95 Jim F. Riggs

96 Lloyd G. Tidwell**
October $\quad 1971$

March $\quad 1972$

May 1973

May 1973

May 1973

October $\quad 1973$

January $\quad 1974$

July $\quad 1974$

April $\quad 1976$

April $\quad 1976$

April $\quad 1976$

April $\quad 1976$

November $\quad 1977$

November 1977

January 1978

January $\quad 1978$

April $\quad 1978$

August $\quad 1978$

March 1979

March 1979

May 1979

May 1979

December 1979

November 1979

March 1980

July 1980

July $\quad 1980$

July $\quad 1980$

March 1981

March 1981

March 1981

March 1981

April $\quad 1981$

June 1981

September 1981

December 1981

December 1981

December 1981

March 1982

May 1982

August 1982

August $\quad 1982$

August $\quad 1982$

September 1982

September 1982

September $\quad 1982$

September 1982

January 1983 


$\begin{aligned} 97 & \text { James Richard Geyer } \\ 98 & \text { Jeffrey Barrett Lawson** } \\ 99 & \text { Brian W. Jarvis** } \\ 100 & \text { Timothy Allen Legel** } \\ 101 & \text { David W. Dahl } \\ 102 & \text { Sharon L. Akemann } \\ 103 & \text { Karen A. Filloon } \\ 104 & \text { Michael L. McCallum** } \\ 105 & \text { Timothy K. Ecker** } \\ 106 & \text { Craig A. Weiner } \\ 107 & \text { Kenneth C. McKinley } \\ 108 & \text { Miles S.Muzio }\end{aligned}$

* Deceased

** Inactive

$\begin{array}{llll}\text { March } & 1983 & 109 & \text { William E. Randby } \\ \text { March } & 1983 & 110 & \text { Troy M. Kimmel, Jr. } \\ \text { April } & 1983 & 111 & \text { Kenneth A. Schulz } \\ \text { April } & 1983 & 112 & \text { Royal L. Norman, Jr. } \\ \text { April } & 1983 & 113 & \text { Michael J. Madson } \\ \text { April } & 1983 & 114 & \text { Wayne G. Mahar** } \\ \text { May } & 1983 & 115 & \text { Renate M. Rao } \\ \text { May } & 1983 & 116 & \text { William S. Schmidt** } \\ \text { February } & 1985 & 117 & \text { Gary Babikian } \\ \text { March } & 1985 & 118 & \text { Joseph M. Rao } \\ \text { July } & 1985 & 119 & \text { David N. Eichorn } \\ \text { August } & 1985 & 120 & \text { Gregory B. Fishel } \\ & & 121 & \text { Paul A. Head } \\ & & 122 & \text { Harold S. Austin, Jr. } \\ & & 123 & \text { James W. Jaggers } \\ & & 124 & \text { Peter V. Chan }\end{array}$

Television Seals of Approval (Alphabetical List of Active Seal Holders)
Adams, John Warren

Addis, Richard A.

Adrian, Barton J.

Albert, Richard E.

Albrecht, Brian A.

Allen, Ron

Alworth, Brian T.

Annen, William $\mathrm{H}$.

Atkinson, Daniel M., Jr.

Aucoin, Kenneth A.

Austin, Martha Lynn

Avalos, Andrew A.

Ban, Raymond J.

Barnes, David P., Jr.

Baron, Robert $\mathrm{O}$.

Bartlett, Joel P., Jr.

Barys, Paul E.

Becker, Robert

Beckman, John F.

Behm, Gordon R.

Belau, Matthew P.

Bernier, Andre Marc

Bernier, John G.

Bevacqua, Thomas J.

Bielski, John T.

Boatman, Loren F.

Bolton, Albert M.

Bono, Michael

Bosley, James W., Sr.

Bostwick, Greg Paul

Bowersox, Stuart M.

Bowman, Daniel Riley

Boylan, Raymond L.

Bray, Steven C.

Brihan, James E.

Brodhead, Gail J.

Brough, Robert C.

Brown, David H.
Brown, George G.

Browne, Steven W.

Brunet, Rene J., III

Burbank, Barry M.

Burns, Glenn Noel

Burse, Tom

Busby, Bryan T.

Buser, Donald C.

Calhoun, Joseph J.

Cannon, Declan P.

Cantore, James M.

Caporizzo, Steven P.

October

1985

October $\quad 1985$

April $\quad 1986$

April $\quad 1986$

November $\quad 1986$

April 1987

April $\quad 1987$

May $\quad 1987$

May $\quad 1987$

May 1987

June 1987

June 1987

December $\quad 1987$

December 1987

March $\quad 1988$

June 1988
Cariello, Frank J. $\quad 478$

Carlson, David C. $\quad 349$

Carmichael, David A. $\quad 417$

Carson, Donald W. 295

Cascione, Steven R. 462

Cavalier, Anthony 314

Cejka, Michael G. $\quad 387$

Chandik, John F. 125

Childress, Elmer M. $\quad 436$

Chuey, Timothy A. $\quad 245$

Cioffi, Joseph $\quad 339$

Clark, Noreen Roberts $\quad 494$

Clark, Thomas N. 495

Clarke, James R. $\quad 406$

Collins, John James 278

Collins, Valerie Voss $\quad 335$

Condella, Vincent D., Jr. $\quad 258$

Coniglio, Martin E. $\quad 469$

Conway, Joseph C. 225

Cooper, Patricia Lynn 479

Copeland, Robert C. 20

Corbin, James M., III 470

Cousins, Paul W. $\quad 185$

Cronise, Walter $\quad 173$

Dahl, David W. 282

Dandrea, Joseph A. 223 
Davies, Jonathan

Davis, Brian D.

Davis, Francis K., Jr.

Day, Robert E., Jr.

Deal, Frank B., Jr.

Deegan, Timothy W.

Dellegatto, Paul N.

Demers, Robert W.

Denardo, Joseph W.

Deprest, Bruce J.

DeZubay, Eric $T$.

Dierks, David G.

Dixon, Robert W.

Dobrowolski, Daniel G.

Dockus, Dale A.

Dooley, John C.

Duisik, David I.

Duncan, James G.

Duranczyk, Edward A., Jr.

Durst, Brian H.

Edwards, Chris S.

Eichorn, David N.

Eisenhood, William O.

Eiser, David S.

Elfervig, Leonard R.

Erwin, Rebecca Ellen

Eubank, Mark E.

Eustis, Allan C.

Evangelista, Mark L.

Evans, William $\mathrm{H}$.

Fairbourne, Michael D.

Fathauer, Theodore F.

Faurot, Richard W.

Fausett, John G.

Feltgen, Dennis W.

Fidler, James C.

Field, Bradford A.

Field, Frank

Field, Storm E.

Filloon, Karen Anne

Finden, Roy F.

Fischer, John C.

Fishel, Gregory B.

Fletcher, Richard R.

Flowers, James B.

Frary, Dennis G.

Fuller, John A.

Ganahl, Jym R.

Gandy, James T.

Gaughan, John C., Jr.

Gelber, Benjamin D.

Gertz, Charles M.

Ghiorse, John T., Jr.

Giddings, Peter F.

Giles, Jimmie D.

Goddard, Richard D.

Gomez, Mario Javier

Graham, Michael H.

Graves, Sharon L.

Gregory, Nicholas J.

Gregory, Robert E.
Griffin, Richard K.

Griffin, Victoria Lee

461

Gross, Paul H.

350

Gross, Todd

217

Grote, Christopher R. $\quad 480$

Guild, David C. 53

Hagar, Paul S. $\quad 178$

Hale, Thomas E. $\quad 280$

Harding, Duane D. 386

Hartman, David R. $\quad 465$

Hartung, Wayne C. 396

Hearst, Ronald A., II 493

Heaton, Jeffrey M. 351

Heppner, Paul O. G. $\quad 455$

Herring, Charles S. 343

Hill, Lawrence D. 256

Hocks, Robert J. $\quad 361$

Hodak, Gerald A. $\quad 154$

Hodges, Dennis John 373

Hoffman, Michael J. ․ 357

Hohmann, Christopher T. 305

Hollett, Shane Richard 415

Hope, John R. 356

Horstmeyer, Steven L. $\quad 322$

Hosler, Charles $\quad 8$

Howes, Ronald L. $\quad 123$

Howl, James M. 177

Huff, Janice Wages 377

Huffines, Bradford T. $\quad 450$

Huntress, David S. $\quad 421$

Jackson, Ronald W. 237

Jaggers, James W. 492

Jensen, Jeffrey T. 445

Johnson, Anthony O'Leary 265

Johnson, Craig A. 162

Johnson, Glenn E. 435

Jones, Lana Cheryl 413

Jones, Valarie Ann 147

Jones, Walton W. 97

Joseph, Paul S. $\quad 85$

Kaderli, Holice H., Jr.

Kalinowski, Bruce E. $\quad 426$

Kamal, William D. 197

Kastor, Neil E. $\quad 247$

Keller, Ted W., Jr. $\quad 408$

Kelly, Terence F. 129

Kierein, Thomas J. 382

Kimmel, Troy M., Jr. 344

Kinnan, Henry W. 3

Kinsey, Carey L., Jr. 253

Klotz, Jeffrey D. $\quad 475$

Knight, Paul G. $\quad 210$

Koch, Kenneth T. 218

Koontz, Mark R. 294

Kovachick, Robert J. 171

Kowal, William R. 116

Kronschnabel, Jeffrey F. 313

Kruhoeffer, Douglas P. 261

Kuster, William E. $\quad 276$

LaNore, Steve C. $\quad 471$

Latham, Jeffrey G. 503 
Lawson, Jeffrey B.

Lawyer, David T.

Leep, Roy L., Jr.

Lennartson, Jay

Leonard, Harvey

Lessens, George D.

Lewis, Norman F.

Ley, Gary W.

Lilley, Tom $\dagger$

Lindmeier, Robert G.

Lizura, Joseph

Loffman, Tom

Loufman, Jon D.

Lozano, Mike A., Jr.

Lyons, Walter A.

Madaus, James L.

Madson, Michael J.

Magnuson, Thomas L.

Maguire, Thaddeus J.

Mahar, Wayne G.

Mahoney, Thomas J.

Malan, John J.

Maly, Daniel D.

Mancuso, Mark

Marsh, David S.

Marshment, Roberta Ann

Marsik, Frank J.

McClellan, Michael H.

McCollister, Daryl W.

McDowell, D. Clay

McLain, Robert W.

McNeely, Donald T.

Mercer, Jack M.

Milford, H. Dale

Milham, Daniel L.

Miller, David M.

Miller-Glahn, Tamara A.

Minshew, Russell F.

Mitchell, Alan L.

Modrick, Michael V.

Morelli, Donald F.

Mroz, Paul J.

Murray, David P.

Muzio, Miles S.

Nefstead, Cyril E.

Nelson, Michael P.

Newman, Stephen R.

Nicholson, Clifford M.

Nolan, Warren D.

Norcross, Brian S.

Norman, Royal L., Jr.

Novak, Donald O.

O'Connell, Kevin M.

O'Donnell, James J.

Oliver, David A.

Ollis, Randall D.

Ott, Armin J.

Owings, Willis B.

Pascal, Neal B.

Paul, Donald J.

Pearl, Edward W.
Penzkowski, Ronald G.

Periconi, Darlene A.

Peterson, William D.

Pettersen, Leon F.

Pope, Daniel W.

Pringle, Henry B.

Prokop, Patrick M.

Provenzano, Andrew C.

Raines, Dallas D.

Rasmussen, Gordon D. 158

Rector, Thomas C. 299

Reeves, Michael G. 211

Reh, William G. $\quad 348$

Reif, James H. 231

Relihan, David J. $\quad 324$

Renner, Jeffrey B. $\quad 488$

Richwien, Barry A. $\quad 269$

Riggio, Robert F. $\quad 497$

Riggs, Jim F. 308

Rixe, Frederick G. $\quad 467$

Roker, $\mathrm{Al} \quad 238$

Rosen, Arnold 393

Rosenzweig, Robert H. $\quad 501$

Ross, Tim $\quad 309$

Rottman, Leon M. 27

Rubin, Eugene M. 251

Rucker, C. Michael 207

Ryan, Robert T. $\quad 79$

Satterfield, Danny E. $\quad 380$

Schaffer, David L. 473

Schellsmidt, Craig S. $\quad 454$

Schmies, Sally P. $\quad 319$

Schmitz, Kurt R. 208

Schnebelt, James J. 293

Schordje, Nicholas D. 367

Schreck, Philip G. $\quad 468$

Schubert, William H. 213

Schulz, Kenneth A. 403

Schwartz, Glenn E. 221

Schwartz, Robert L. $\quad 212$

Schwarz, Philip S. $\quad 409$

Schwoegler, Bruce W. $\quad 189$

Sealls, Alan R. $\quad 486$

Sebastian, Norman C. 381

Shapiro, Howard E. 146

Shattuck, Wayne C. 214

Shore, Gary 155

Shutt, Charles T. 498

Sill, Suzanne D. $\quad 487$

Sillars, Malcolm P. 285

Silvestri, Paul J. $\quad 325$

Sisto, F. Calvin 192

Skilling, Thomas E., III $\quad 119$

Slesick, Leonard M. $\quad 57$

Smith, Dennis L. 215

Smith, James E. $\quad 44$

Smith, Michael R. $\quad 130$

Sobel, Joseph P. 103

Sonen, Heidi E. $\quad 502$

Stachak, Stanley J. 222

Stanley, Phillip P. $\quad 485$ 
Steffen, William J.

Stewart, James F.

Stinnett, Lorraine

Swails, Terry L.

Taft, Jerome B., II

Tasselmyer, Thomas $\mathrm{M}$.

Taylor, Norman C.

Teller, Merril D.

Textor, Theodore E.

Thompson, Chris D.

Thompson, Michael L.

Towne, David K.

Triemstra, Roger L.

True, Dan J.

Umpenhour, Charles M.

Voss, Valerie Collins

Volkman, Harry A.

Wappler, Harry V.

Waunsch, John G.

Weaver, Robert

Webre, Byron $\mathrm{K}$.

Wegner, James R.

Weingarten, Andrew L.
Weiss, Carl E., Jr. 270

Weiss, Fred W.

Wendel, John M.

Westbrook, Don R.

Westerlage, Keith C.

Whitelaw, Phil

Whitford, David C.

Williams, Kevin D.

Willing, John R.

Wills, Thomas G.

Winfield, Alan S.

Winston, Wayne G.

Winterling, George A.

Wood, Glenn C.

Woodford, Richard A.

Woods, Craig J.

Woods, Donald K.

Wooldridge, John M.

Yaros, Ronald A.

Zabrecky, George S.

Zabrecky, Robert E.

Zaffino, Matthew

Zeliff, William M.

Zunke, Dale N.
120

317

144

439

312

418

340

232

80

419

95

42

271

452

113

150

420

272

431

376

84

202

\section{Television Seal Holders (Listed by Certificate Number)}

$\begin{array}{rll}1 & \text { Francis K. Davis, Jr. } & \text { Honorary } \\ 2 & \text { Kenneth H. Jehn* } & \text { Honorary } \\ 3 & \text { Henry W. Kinnan } & \text { Honorary } \\ 4 & \text { Milton F. Barlow** } \\ 5 & \text { Warren A. Culbertson** } \\ 6 & \text { Bob Thomas** } \\ 7 & \text { Robert L. Hendrick** } \\ 8 & \text { Charles Hosler } \\ 9 & \text { George M. Howe** } \\ 10 & \text { Roy L. Leep } \\ 11 & \text { James M. Macdonald, Jr. } * * \\ 12 & \text { Frederick P. Ostby, Jr.** } \\ 13 & \text { Donald E. Kent** } \\ 14 & \text { Peter M. Kuhn** } \\ 15 & \text { Milton A. Strauss** } \\ 16 & \text { Gordon B. Weir* } \\ 17 & \text { Joe Hines Wolters** } \\ 18 & \text { Cecil C. Carrier** } \\ 19 & \text { John C. Capell** } \\ 20 & \text { Robert C. Copeland } \\ 21 & \text { D. Clay McDowell } \\ 22 & \text { Albert E. Boyer, Jr.** } \\ 23 & \text { Harry A. Volkman } \\ 24 & \text { Howard M. Frazier** } \\ 25 & \text { H. Dale Milford } \\ 26 & \text { Frank H. Forrester** } \\ 27 & \text { Leon M. Rottman } \\ & \end{array}$

$\begin{array}{ll}\text { January } & 1959 \\ \text { January } & 1959 \\ \text { January } & 1959 \\ \text { January } & 1960 \\ \text { January } & 1960 \\ \text { January } & 1960 \\ \text { January } & 1960 \\ \text { January } & 1960 \\ \text { January } & 1960 \\ \text { January } & 1960 \\ \text { January } & 1960 \\ \text { January } & 1960 \\ \text { January } & 1960 \\ \text { January } & 1960 \\ \text { January } & 1960 \\ \text { January } & 1960 \\ \text { January } & 1960 \\ \text { January } & 1960 \\ \text { April } & 1960 \\ \text { April } & 1960 \\ \text { April } & 1960 \\ \text { October } & 1960 \\ \text { October } & 1960 \\ \text { October } & 1960 \\ \text { November } & 1960 \\ \text { November } & 1960 \\ \text { December } & 1960\end{array}$

* Deceased

** Inactive

\begin{tabular}{|c|c|c|c|}
\hline 28 & James C. Fidler & January & 1961 \\
\hline 29 & William Crawford* & April & 1961 \\
\hline 30 & Joseph W. Denardo & April & 1961 \\
\hline 31 & Verl D. Dotson** & April & 1961 \\
\hline 32 & Charles F. Thomas** & August & 1961 \\
\hline 33 & Nash C. Roberts, Jr.** & November & 1961 \\
\hline 34 & Howard E. Reiquam** & November & 1961 \\
\hline 35 & Norman J. MacDonald** & January & 1962 \\
\hline 36 & Harry C. Zimmerman** & February & 1962 \\
\hline 37 & Norman K. Wagner** & May & 1962 \\
\hline 38 & Robert E. Amsberry** & May & 1962 \\
\hline 39 & Robert E. Lynott** & June & 1962 \\
\hline 40 & Howard H. Hanks, Jr.* & June & 1962 \\
\hline 41 & Roland R. Kessler** & June & 1962 \\
\hline 42 & George A. Winterling & May & 1963 \\
\hline 43 & Loren F. Boatman & July & 1963 \\
\hline 44 & James E. Smith & November & 1963 \\
\hline 45 & Richard D. Goddard & July & 1964 \\
\hline 46 & Charles W. Stump* & July & 1964 \\
\hline 47 & Albert W. Duckworth** & September & 1964 \\
\hline 48 & Clifford H. Watkins** & March & 1965 \\
\hline 49 & Hubert D. Bagley** & March & 1965 \\
\hline 50 & George W. Sickels, Jr.** & May & 1965 \\
\hline 51 & John W. Hambleton** & July & 1966 \\
\hline 52 & Frank Field & July & 1966 \\
\hline 53 & David C. Guild & September & 1966 \\
\hline 54 & Seth D. Kemble** & February & 1967 \\
\hline 55 & Conrad L. Johnson** & April & 1967 \\
\hline 56 & Horace W. Meredith** & May & 1967 \\
\hline 57 & Leonard M. Slesick & May & 1967 \\
\hline 58 & Robert F. Zames** & May & 1967 \\
\hline
\end{tabular}


Vol. 69, No. 8, August 1988

59 Frederick J. Norman**

60 W. Paul Catoe**

61 John H. Cromwell**

62 James D. Williams**

63 Ronald L. Godbey**

64 Arthur C. Roberts**

65 Boyd E. Quate**

66 Tony Sands**

67 Robert Weaver

68 Jehu D. Ashmore**

69 Robert Kudzma**

70 Charles Melvin Gertz

71 Robert E. Wademan**

72 Joel N. Myers**

73 John J. Cahir**

74 John T. Ghiorse, Jr.

75 Richard G. Andrews**

76 Charles M. Umpenhour

77 William N. Seiler**

78 George R. Wooten, Jr.**

79 Robert T. Ryan

80 Thomas G. Wills

81 Roger L. Triemstra

82 Elliot Abrams**

83 Sidney O. Barnard**

84 William M. Zeliff

85 Paul S. Joseph

86 Charles Middleton**

87 Douglas B. Cargill**

88 Harry J. Green**

89 Mark E. Eubank

90 Robert E. Zabrecky

91 William S. Thorpe**

92 George R. Fischbeck**

93 John T. McMurray**

94 H. Joe Witte**

95 Wayne G. Winston

96 June Bacon-Bercey**

97 Walton W. Jones

98 Walter A. Lyons

99 Eric A. Meindl**

100 John F. Beckman

101 Thomas J. Mahoney

102 Mark H. Schumacher**

103 Joseph P. Sobel

104 Allen H. Motew**

105 Virginia J. Bigler-Engler**

106 Jym R. Ganahl

107 Ross J. Dixon, Jr.**

108 William B. Hovey**

109 John A. Ebert**

110 Patrick B. Moore**

111 Allan C. Eustis

112 Richard E. Albert

113 Craig J. Woods

114 Winston H. Jervis, Jr.**

115 James J. O'Donnell

116 William R. Kowal

\begin{tabular}{|c|c|c|c|}
\hline September & 1967 & 117 & Norman C. Taylor \\
\hline September & 1967 & 118 & Russell G. DuBuc** \\
\hline March & 1968 & 119 & Thomas E. Skilling III \\
\hline April & 1968 & 120 & Fred W. Weiss \\
\hline September & 1968 & 121 & Robert D. Welti** \\
\hline September & 1968 & 122 & Russell F. Minshew \\
\hline September & 1968 & 123 & Ronald L. Howes \\
\hline February & 1969 & 124 & Norman F. Lewis \\
\hline July & 1969 & 125 & John F. Chandik \\
\hline August & 1969 & 126 & Kenneth R. Rainey** \\
\hline August & 1969 & 127 & John R. Bradshaw** \\
\hline October & 1969 & 128 & Peter F. Giddings \\
\hline October & 1969 & 129 & Terence F. Kelly \\
\hline October & 1969 & 130 & Michael R. Smith \\
\hline October & 1969 & 131 & Dennis W. Feltgen \\
\hline October & 1969 & 132 & Cecil S. Keen** \\
\hline October & 1969 & 133 & Kenneth B. McCool** \\
\hline December & 1969 & 134 & John B. Walls. $* *$ \\
\hline July & 1970 & 135 & Alan S. Mitleider** \\
\hline September & 1970 & 136 & David S. Marsh \\
\hline January & 1971 & 137 & Paul E. Barys \\
\hline May & 1971 & 138 & James F. Pass** \\
\hline May & 1971 & 139 & Raymond L. Boylan \\
\hline May & 1971 & 140 & Joel P. Bartlett, Jr. \\
\hline July & 1971 & 141 & Robert J. Roseman** \\
\hline July & 1971 & 142 & Donald S. Noe** \\
\hline July & 1971 & 143 & Richard D. McLaughlin** \\
\hline July & 1971 & 144 & Don R. Westbrook \\
\hline October & 1971 & 145 & Charles E. Levy** \\
\hline November & 1971 & 146 & Howard E. Shapiro** \\
\hline March & 1972 & 147 & Valarie Ann Jones \\
\hline March & 1972 & 148 & James Lee Menard** \\
\hline March & 1972 & 149 & David P. Murray \\
\hline May & 1972 & 150 & Donald K. Woods \\
\hline September & 1972 & 151 & Richard W. Faurot \\
\hline November & 1972 & 152 & Jimmie D. Giles \\
\hline December & 1972 & 153 & Richard J. Katz** \\
\hline December & 1972 & 154 & Gerald A. Hodak \\
\hline December & 1972 & 155 & Gary Shore \\
\hline December & 1972 & 156 & Roy Fred Finden \\
\hline December & 1972 & 157 & Richard J. Mancini** \\
\hline April & 1973 & 158 & Gordon D. Rasmussen \\
\hline October & 1973 & 159 & Fred J. Jenkins** \\
\hline October & 1973 & 160 & Richard R. Fletcher \\
\hline October & 1973 & 161 & Vincent M. Miller** \\
\hline October & 1973 & 162 & Craig A. Johnson \\
\hline December & 1973 & 163 & James R. Little** \\
\hline December & 1973 & 164 & Robert Paul Soper** \\
\hline April & 1974 & 165 & Wayne M. Jenkins** \\
\hline April & 1974 & 166 & Armin J. Ott \\
\hline March & 1974 & 167 & Dale G. Noah** \\
\hline May & 1974 & 168 & Jerome B. Taft II \\
\hline July & 1974 & 169 & Edward J. Ring** \\
\hline July & 1974 & 170 & Theodore F. Fathauer \\
\hline July & 1974 & 171 & Robert J. Kovachick \\
\hline July & 1974 & 172 & Robert W. Jacobson, Jr.** \\
\hline July & 1974 & 173 & Walter Cronise \\
\hline \multirow[t]{3}{*}{ July } & 1974 & 174 & Michael D. Fairbourne \\
\hline & & 175 & Dallas D. Raines \\
\hline & & $\begin{array}{l}176 \\
177\end{array}$ & Mike A. Lozano, Jr. \\
\hline
\end{tabular}

\begin{tabular}{|c|c|}
\hline July & 1974 \\
\hline January & 1975 \\
\hline January & 1975 \\
\hline January & 1975 \\
\hline January & 1975 \\
\hline January & 1975 \\
\hline March & 1975 \\
\hline April & 1975 \\
\hline April & 1975 \\
\hline April & 1975 \\
\hline May & 1975 \\
\hline June & 1975 \\
\hline July & 1975 \\
\hline August & 1975 \\
\hline October & 1975 \\
\hline January & 1976 \\
\hline April & 1976 \\
\hline April & 1976 \\
\hline April & 1976 \\
\hline April & 1976 \\
\hline July & 1976 \\
\hline July & 1976 \\
\hline January & 1977 \\
\hline January & 1977 \\
\hline January & 1977 \\
\hline November & 1977 \\
\hline November & 1977 \\
\hline January & 1978 \\
\hline January & 1978 \\
\hline January & 1978 \\
\hline January & 1978 \\
\hline January & 1978 \\
\hline January & 1978 \\
\hline April & 1978 \\
\hline April & 1978 \\
\hline April & 1978 \\
\hline April & 1978 \\
\hline July & 1978 \\
\hline July & 1979 \\
\hline July & 1978 \\
\hline July & 1978 \\
\hline July & 1978 \\
\hline July & 1978 \\
\hline July & 1978 \\
\hline July & 1978 \\
\hline July & 1978 \\
\hline July & 1978 \\
\hline July & 1978 \\
\hline July & 1978 \\
\hline August & 1978 \\
\hline August & 1978 \\
\hline August & 1978 \\
\hline August & 1978 \\
\hline October & 1978 \\
\hline October & 1978 \\
\hline October & 1978 \\
\hline October & 1978 \\
\hline October & 1978 \\
\hline October & 1978 \\
\hline October & 1978 \\
\hline March & 1979 \\
\hline
\end{tabular}

* Deceased
** Inactive 


\begin{tabular}{|c|c|}
\hline 178 & Paul S. Hagar \\
\hline 179 & William O. Eisenhood \\
\hline 180 & Robert L. O’Wril* \\
\hline 181 & H. Michael Mogil** \\
\hline 182 & Robert W. Dixon \\
\hline 183 & Leonard R. Elfervig \\
\hline 184 & Patrick M. Prokop \\
\hline 185 & Paul W. Cousins \\
\hline 186 & Richard C. Przywarty** \\
\hline 187 & Dan J. True \\
\hline 188 & Merril D. Teller \\
\hline 189 & Bruce W. Schwoegler \\
\hline 190 & Jeffrey P. Walker** \\
\hline 191 & Richard R. Warren** \\
\hline 192 & F. Calvin Sisto \\
\hline 193 & William Dale Peterson \\
\hline 194 & Jeffrey B. Gallant** \\
\hline 195 & Keith Eichner** \\
\hline 196 & Robert W. McLain \\
\hline 197 & William D. Kamal \\
\hline 198 & Michael H. Graham \\
\hline 199 & Arthur E. Hornberger, Jr.** \\
\hline 200 & Daniel D. Maly \\
\hline 201 & Warren D. Nolan \\
\hline 202 & Dale N. Zunke \\
\hline 203 & Daniel M. Atkinson, Jr. \\
\hline 204 & Barry M. Burbank \\
\hline 205 & David I. Duisik \\
\hline 206 & Mark S. Russell** \\
\hline 207 & C. Michael Rucker \\
\hline 208 & Kurt R. Schmitz \\
\hline 209 & Harry V. Wappler \\
\hline 210 & Paul G. Knight \\
\hline 211 & Michael G. Reeves \\
\hline 212 & Robert L. Schwartz \\
\hline 213 & William H. Schubert \\
\hline 214 & Wayne C. Shattuck \\
\hline 215 & Dennis L. Smith \\
\hline 216 & Benjamin D. Gelber \\
\hline 217 & Todd Gross \\
\hline 218 & Kenneth T. Koch \\
\hline 219 & John J. Malan \\
\hline 220 & Jack M. Mercer \\
\hline 221 & Glenn E. Schwartz \\
\hline 222 & Stanley J. Stachak \\
\hline 223 & Joseph A. Dandrea \\
\hline 224 & Thomas L. Magnuson \\
\hline 225 & Joseph C. Conway \\
\hline 226 & Brian S. Norcross \\
\hline 227 & Roberta Ann Marshment \\
\hline 228 & Harvey Leonard \\
\hline 229 & James L. Madaus \\
\hline 230 & Lawrence J. Mulholland** \\
\hline 231 & James H. Reif \\
\hline 232 & John Robert Willing \\
\hline 233 & William H. Annen \\
\hline 234 & John D. Flanders** \\
\hline
\end{tabular}

\begin{tabular}{|c|c|c|c|}
\hline March & 1979 & 235 & James Brian Flowers \\
\hline March & 1979 & 236 & Kevin M. O’Connell \\
\hline March & 1979 & 237 & Ronald W. Jackson \\
\hline April & 1979 & 238 & Albert L. Roker \\
\hline April & 1979 & 239 & James W. Bosley, Sr. \\
\hline April & 1979 & 240 & Wayne P. Chandler** \\
\hline April & 1979 & 241 & Daniel G. Dobrowolski \\
\hline April & 1979 & 242 & Robert E. Gregory \\
\hline April & 1979 & 243 & Thomas Loffman \\
\hline April & 1979 & 244 & Nancy A. Russo Chapman \\
\hline April & 1979 & 245 & Timothy A. Chuey \\
\hline April & 1979 & 246 & Dennis G. Frary \\
\hline April & 1979 & 247 & Neil E. Kastor \\
\hline July & 1979 & 248 & Donald T. McNeely \\
\hline July & 1979 & 249 & Michael P. Nelson \\
\hline July & 1979 & 250 & Donald O. Novak \\
\hline September & 1979 & 251 & Eugene M. Rubin \\
\hline September & 1979 & 252 & Jocelyn K. White** \\
\hline October & 1979 & 253 & Carey L. Kinsey, Jr. \\
\hline October & 1979 & 254 & David K. Towne \\
\hline November & 1979 & 255 & Richard A. Addis \\
\hline November & 1979 & 256 & Lawrence D. Hill \\
\hline November & 1979 & 257 & James G. Duncan \\
\hline November & 1979 & 258 & Vincent D. Condella, Jr. \\
\hline November & 1979 & 259 & Glenn Noel Burns \\
\hline March & 1980 & 260 & Louis K. McNally III** \\
\hline March & 1980 & 261 & Douglas P. Kruhoeffer \\
\hline March & 1980 & 262 & Robert Becker \\
\hline March & 1980 & 263 & Stuart M. Bowersox \\
\hline April & 1980 & 264 & Gregory B. Fishel \\
\hline May & 1980 & 265 & Anthony O'Leary Johnson \\
\hline July & 1980 & 266 & Mark L. Nichols** \\
\hline December & 1980 & 267 & Neal B. Pascal \\
\hline December & 1980 & 268 & Donald J. Paul \\
\hline December & 1980 & 269 & Barry A. Richwien \\
\hline December & 1980 & 270 & Carl E. Weiss, Jr. \\
\hline December & 1980 & 271 & Glenn C. Wood \\
\hline December & 1980 & 272 & Ronald A. Yaros \\
\hline March & 1981 & 274 & John G. Bernier \\
\hline March & 1981 & 275 & Clifford M. Nicholson \\
\hline March & 1981 & 276 & William E. Kuster \\
\hline March & 1981 & 277 & Frank B. Deal, Jr. \\
\hline March & 1981 & 278 & John James Collins \\
\hline March & 1981 & 279 & John J. Campbell** \\
\hline March & 1981 & 280 & Thomas E. Hale \\
\hline April & 1981 & 281 & Robert O. Baron \\
\hline April & 1981 & 282 & David W. Dahl \\
\hline June & 1981 & 283 & Willis Bryan Owings \\
\hline June & 1981 & 284 & Daniel L. Milham \\
\hline June & 1981 & 285 & Malcolm P. Sillars \\
\hline August & 1981 & 286 & Andre Marc Bernier \\
\hline August & 1981 & 287 & John T. Bielski \\
\hline August & 1981 & 288 & Edward A. Duranczyk, Jr. \\
\hline August & 1981 & 289 & William C. Keneely** \\
\hline August & 1981 & 290 & Michael V. Modrick** \\
\hline September & 1981 & 291 & Cyril E. Nefstead \\
\hline \multirow[t]{5}{*}{ September } & 1981 & 292 & Leon F. Pettersen \\
\hline & & 293 & James J. Schnebelt \\
\hline & & 294 & Mark R. Koontz \\
\hline & & 295 & Donald W. Carson \\
\hline & & 296 & David \\
\hline
\end{tabular}

\begin{tabular}{|c|c|}
\hline September & 1981 \\
\hline September & 1981 \\
\hline October & 1981 \\
\hline November & 1981 \\
\hline December & 1981 \\
\hline December & 1981 \\
\hline December & 1981 \\
\hline December & 1981 \\
\hline January & 1982 \\
\hline January & 1982 \\
\hline March & 1982 \\
\hline March & 1982 \\
\hline March & 1982 \\
\hline March & 1982 \\
\hline March & 1982 \\
\hline March & 1982 \\
\hline March & 1982 \\
\hline May & 1982 \\
\hline May & 1982 \\
\hline June & 1982 \\
\hline June & 1982 \\
\hline February & 1982 \\
\hline June & 1982 \\
\hline June & 1982 \\
\hline June & 1982 \\
\hline June & 1982 \\
\hline June & 1982 \\
\hline August & 1982 \\
\hline August & 1982 \\
\hline August & 1982 \\
\hline August & 1982 \\
\hline August & 1982 \\
\hline August & 1982 \\
\hline August & 1982 \\
\hline August & 1982 \\
\hline August & 1982 \\
\hline August & 1982 \\
\hline August & 1982 \\
\hline September & 1982 \\
\hline September & 1982 \\
\hline September & 1982 \\
\hline September & 1982 \\
\hline September & 1982 \\
\hline September & 1982 \\
\hline September & 1982 \\
\hline September & 1982 \\
\hline September & 1982 \\
\hline September & 1982 \\
\hline September & 1982 \\
\hline September & 1982 \\
\hline January & 1983 \\
\hline January & 1983 \\
\hline January & 1983 \\
\hline January & 1983 \\
\hline January & 1983 \\
\hline January & 1983 \\
\hline January & 1983 \\
\hline January & 1983 \\
\hline January & 1983 \\
\hline March & 1983 \\
\hline March & 1983 \\
\hline
\end{tabular}


297 Mark Mancuso

298 Randall D. Ollis

299 Thomas C. Rector

300 Terry L. Swails

301 Nicholas J. Gregory

302 Chuck F. Wiese**

303 Barton J. Adrian

304 Raymond J. Ban

305 Christopher T. Hohmann

306 Gary W. Ley

307 Albert M. Bolton

308 Jim F. Riggs

309 Tim Ross

310 Donald C. Buser

311 Miles S. Muzio

312 Phil Whitelaw

313 Jeffrey F. Kronschnabel

314 Anthony Cavalier

315 Dale Alan Dockus

316 David N. Eichorn

317 John M. Wendel

318 John C. Dooley

319 Sally P. Schmies

320 Michael L. Thompson

321 Steven W. Browne

322 Steven L. Horstmeyer

323 Stephen M. Letro**

324 David J. Relihan

325 Paul J. Silvestri

326 Storm E. Field

327 James E. Brihan

328 Karen Anne Filloon

329 Timothy W. Deegan

330 Henry B. Pringle

331 Jonathan Davies

332 Robert Arnold**

333 Donald L. Edwards**

334 Bradford A. Field

335 Valerie Voss Collins

336 John C. Fischer

337 Gail J. Brodhead

338 Joseph J. Calhoun

339 Joseph Cioffi

340 Kevin D. Williams

341 David T. Lawyer

342 Chris D. Thompson

343 Charles S. Herring

344 Troy M. Kimmel, Jr.

345 Jeffrey B. Lawson

346 Donald K. Chilo**

347 Lorraine Stinnett

348 William G. Reh

349 David C. Carlson

350 Paul H. Gross

351 Jeffrey M. Heaton

352 Brian D. Davis

353 David P. Barnes, Jr.

* Deceased

** Inactive

\begin{tabular}{|c|c|c|c|c|c|}
\hline March & 1983 & 354 & David H. Brown & February & 1985 \\
\hline March & 1983 & 355 & Michael H. McClellan & February & 1985 \\
\hline March & 1983 & 356 & John R. Hope & February & 1985 \\
\hline March & 1983 & 357 & Michael J. Hoffman & February & 1985 \\
\hline March & 1983 & 358 & Robert E. Day, Jr. & February & 1985 \\
\hline March & 1983 & 359 & Rufus R. Hafer** & February & 1985 \\
\hline March & 1983 & 360 & Ron Allen & February & 1985 \\
\hline March & 1983 & 361 & Robert J. Hocks & February & 1985 \\
\hline March & 1983 & 362 & David A. Oliver & February & 1985 \\
\hline March & 1983 & 363 & Joseph A. Pietrowicz** & March & 198 \\
\hline May & 1983 & 364 & John A. Fuller & March & 1985 \\
\hline May & 1983 & 365 & Rebecca Ellen Erwin & March & 1985 \\
\hline June & 1983 & 366 & James P. Roemer** & March & 1985 \\
\hline June & 1983 & 367 & Nicholas D. Schordje & March & 1985 \\
\hline June & 1983 & 368 & Edward W. Pearl & May & 1985 \\
\hline June & 1983 & 369 & James T. Gandy & June & 1985 \\
\hline July & 1983 & 370 & Mario J. Gomez & June & 1985 \\
\hline July & 1983 & 371 & Daniel Riley Bowman & May & 198 \\
\hline July & 1983 & 372 & Greg Paul Bostwick & June & 198 \\
\hline August & 1983 & 373 & Dennis John Hodges & June & 1985 \\
\hline August & 1983 & 374 & Eric T. DeZubay & June & 198 \\
\hline August & 1983 & 375 & Paul J. Mroz & June & 198 \\
\hline August & 1983 & 376 & Matthew Zaffino & June & 198 \\
\hline September & 1983 & 377 & Janice Wages Huff & July & 198 \\
\hline September & 1983 & 378 & Richard K. Griffin & July & 198 \\
\hline September & 1983 & 379 & Stephen R. Newman & July & 198 \\
\hline September & 1983 & 380 & Danny E. Satterfield & July & 198 \\
\hline September & 1983 & 381 & Norman C. Sebastian & July & 198 \\
\hline September & 1983 & 382 & Thomas J. Kierein & September & 198 \\
\hline October & 1983 & 383 & George D. Lessens & September & 198 \\
\hline October & 1983 & 384 & Joseph Lizura & September & 198 \\
\hline October & 1983 & 385 & Royal L. Norman, Jr. & September & 198 \\
\hline November & 1983 & 386 & Duane D. Harding & October & 198 \\
\hline November & 1983 & 387 & Michael G. Cejka & October & 198 \\
\hline November & 1983 & 388 & Jay Lennartson & October & 198 \\
\hline November & 1983 & 390 & Steven P. Caporizzo & October & 198 \\
\hline November & 1983 & 391 & William H. Evans & October & 1985 \\
\hline December & 1983 & 392 & Wayne G. Mahar & October & 198 \\
\hline December & 1983 & 393 & Arnold Rosen & October & 198 \\
\hline February & 1984 & 394 & Herbert Eugene Stevens & October & 198 \\
\hline February & 1984 & 395 & David M. Miller & October & 198 \\
\hline June & 1984 & 396 & Wayne Carr Hartung & November & 198 \\
\hline June & 1984 & 397 & Robert G. Lindmeier & November & 198 \\
\hline June & 1984 & 398 & Daniel W. McCarthy** & December & 198 \\
\hline August & 1984 & 399 & Thaddeus J. Maguire & December & 198 \\
\hline August & 1984 & 400 & Andrew L. Weingarten & December & 198 \\
\hline September & 1984 & 401 & Robert C. Brough & December & 198 \\
\hline September & 1984 & 402 & Chris S. Edwards & January & 198 \\
\hline September & 1984 & 403 & Kenneth A. Schulz & January & 198 \\
\hline September & 1984 & 404 & Brian A. Albrecht & February & 198 \\
\hline September & 1984 & 405 & Michael Bono & February & 198 \\
\hline September & 1984 & 406 & James R. Clarke & February & 198 \\
\hline December & 1984 & 407 & Andrew A. Avalos & March & 198 \\
\hline December & 1984 & 408 & Ted William Keller, Jr. & March & 198 \\
\hline December & 1984 & 409 & Philip S. Schwarz & March & 198 \\
\hline December & 1984 & 410 & John Warren Adams & March & 198 \\
\hline \multirow[t]{5}{*}{ February } & 1985 & 411 & James Robert Wegner & March & 198 \\
\hline & & 412 & William J. Steffen & April & 198 \\
\hline & & 413 & Lana Cheryl Jones & April & \\
\hline & & 414 & Sharon L. Graves & May & \\
\hline & & 415 & Shane Richard Hollett & May & \\
\hline
\end{tabular}


416 Donald F. Morelli

417 David A. Carmichael

418 David C. Whitford

419 Alan S. Winfield

420 John M. Wooldridge

421 David S. Huntress

422 Tom Lilley $\dagger$

423 John G. Waunsch

424 Matthew P. Belau

425 Bryan T. Busby

426 Bruce E. Kalinowski

427 Ronald G. Penzkowski

428 Mark B. Rosenthal**

429 Sharon Louise Akemann**

430 Thomas M. Tasselmyer

431 George S. Zabrecky

432 Byron K. Webre

433 Bruce J. Deprest

434 Holice H. Kaderli, Jr.

435 Glenn E. Johnson

436 Elmer M. Childress

437 Thomas J. Bevacqua

438 Robert W. Cameron**

439 Keith Charles Westerlage

440 James F. Stewart

441 Andrew C. Provenzano

442 Michael S. Rampy**

443 John Charles Gaughan, Jr.

444 Gordon R. Behm

445 Jeffrey T. Jensen

446 William S. Schmidt**

447 Tom Burse

448 Brian H. Durst

449 Martha Lynn Austin

450 Bradford T. Huffines

451 Rene J. Brunet III

452 Richard A. Woodford

453 Robert W. Demers

454 Craig S. Schellsmidt

455 Paul O. G. Heppner

456 John R. Boston**

$\begin{array}{ll}\text { May } & 1986 \\ \text { June } & 1986 \\ \text { June } & 1986 \\ \text { July } & 1986 \\ \text { July } & 1986 \\ \text { August } & 1986 \\ \text { August } & 1986 \\ \text { September } & 1986 \\ \text { October } & 1986 \\ \text { October } & 1986 \\ \text { October } & 1986 \\ \text { October } & 1986 \\ \text { October } & 1986 \\ \text { November } & 1986 \\ \text { December } & 1986 \\ \text { December } & 1986 \\ \text { February } & 1987 \\ \text { February } & 1987 \\ \text { February } & 1987 \\ \text { February } & 1987 \\ \text { March } & 1987 \\ \text { April } & 1987 \\ \text { April } & 1987 \\ \text { April } & 1987 \\ \text { April } & 1987 \\ \text { April } & 1987 \\ \text { April } & 1987 \\ \text { April } & 1987 \\ \text { May } & 1987 \\ \text { May } & 1987 \\ \text { May } & 1987 \\ \text { May } & 1987 \\ \text { May } & 1987 \\ \text { June } & 1987 \\ \text { October } & 1987 \\ \text { October } & 1987 \\ \text { October } & 1987 \\ \text { October } & 1987 \\ \text { October } & 1987 \\ \text { October } & 1987 \\ \text { October } & 1987\end{array}$

* Deceased

** Inactive

$\dagger$ Non-member
457 John A. Cessarich**

458 Theodore E. Textor

459 Daryl W. McCollister

460 Brian T. Alworth

461 Victoria Lee Griffin

462 Steven R. Cascione

463 Kenneth A. Aucoin

464 John G. Fausett

465 David R. Hartman

466 William E. Randby**

467 Frederick G. Rixe

468 Philip G. Schreck

469 Martin E. Coniglio

470 James M. Corbin III

471 Steve C. LaNore

472 Daniel W. Pope

473 David L. Schaffer

474 James M. Cantore

475 Jeffrey D. Klotz

476 Steven C. Bray

477 Declan P. Cannon

478 Frank J. Cariello

479 Patricia Lynn Cooper

480 Christopher R. Grote

481 Jon D. Loufman

482 Michael J. Madson

483 Frank J. Marsik

484 Darlene A. Periconi

485 Phillip P. Stanley

486 Alan R. Sealls

487 Suzanne D. Sill

488 Jeffrey B. Renner

489 Paul N. Dellegatto

490 David G. Dierks

491 Mark L. Evangelista

492 James W. Jaggers

493 Ronald A. Hearst II

494 Noreen Roberts Clark

495 Thomas N. Clark

496 Alan L. Mitchell

497 Robert F. Riggio

498 Charles T. Shutt

499 Tamara A. Miller-Glahn

500 George G. Brown

501 Robert H. Rosenzweig

502 Heidi E. Sonen

503 Jeffrey G. Latham
October

1987

November

December

December

December

December

December

December

December

December

December

December

January

January

January

January

January

January

January

February

February

February

February

February

February

February

February

February

February

February

February

February

March

March

March

March

March

April

April

April

April

April

May

May

May

June

June
1987

1987

1987

1987

1987

1987

1987

1987

1987

1987

1987

1988

1988

1988

1988

1988

1988

1988

1988

1988

1988

1988

1988

1988

1988

1988

1988

1988

1988

1988

1988

1988

1988

1988

1988

1988

1988

1988

1988

1988

1988

1988

1988

1988

1988

1988 